\title{
Mucin producing microfollicular adenoma of the thyroid
}

\author{
CLAUDE RIGAUD, F PELTIER, * WV BOGOMOLETZ
}

From the Laboratoires d'anatomie pathologique, Institut Jean Godinot, Reims, and ${ }^{*}$ Centre Hospitalier Général, St Quentin, France

SUMMARY An unusual case of a mucin secreting benign microfollicular adenoma of the thyroid in a 30 year old euthyroid woman is reported. Histologically, the lesion was characterised by follicular cells with the appearance of signet ring cells. Histochemistry showed the mucin content of these cells to consist uniformly of sulphated acid mucins; positive thyroglobulin immunostaining was also shown. The published work on primary mucin secreting tumours of the thyroid gland is reviewed. Dual differentiation is thought to be responsible for combined mucin secretion and hormone production in this type of neoplasm.

Primary tumours of the thyroid gland producing mucin are extremely rare and their histogenesis is controversial. Few cases of primary mucinous or mucoepidermoid carcinomas of the thyroid have been reported..$^{1-4}$ Mucin production has also been described in some cases of medullary carcinoma of the thyroid. ${ }^{5-7}$ Recently, the first case of a mucin secreting benign adenoma of the thyroid was reported. $^{8}$

We report here what we believe to be the second recorded case of mucin secreting benign microfollicular adenoma of the thyroid gland. We present the results of our immunocytochemical and mucin histochemical study of this peculiar tumour and we discuss different aspects of its histogenesis.

\section{Case report}

A euthyroid 30 year old woman presented with a goitre in January 1981. Physical examination disclosed a palpable solitary nodule in the right lobe of the thyroid. The nodule appeared as a cold area on the thyroid scintiscan, while the remainder of the gland showed normal uptake. Clinical and laboratory investigations were otherwise unremarkable. There was no history of neoplastic disease in the thyroid or elsewhere. No malignant cells were identified in the smears from a fine needle aspiration specimen of the nodule. The patient received a 6 month trial course of thyroxine, which produced little decrease in size of the nodule. A right hemithyroidectomy was carried out in October
1981. At the time of operation frozen section was reported as carcinoma and a total thyroidectomy was performed. The patient made an uneventful postoperative recovery and was discharged. She has been examined at regular intervals since her operation. The last follow up examination was in February 1984 and the patient was then in good health.

\section{PATHOLOGY}

Macroscopically, the right lobe of the thyroid contained a well circumscribed nodule measuring $2 \mathrm{~cm}$ in diameter. The remainder of the right lobe as well as the isthmus and left lobe of the thyroid showed no gross abnormality.

Histologically, the nodule was also well circumscribed and almost entirely enclosed within a thin fibrous capsule (Fig. 1). The nodule consisted predominantly of nests and columns of closely packed, pale staining cells; each showed an eccentrically placed nucleus and a large central vacuole (Fig. 2). The overall appearances of these cells suggested signet ring cells. Interspersed among the pale vacuolated cells were appreciable numbers of small thyroid follicles containing colloid material. The nuclei of the pale vacuolated cells as well as those lining the small follicles appeared cytologically bland and showed no evidence of atypia or abnormal mitotic activity. The Grimelius-silver technique and diazoalkaline and Masson-Fontana methods were negative for argentaffin and argyrophil granules. The nodule also contained some fibrous stroma which stained positively for collagen with Van Gieson and negatively for amyloid with Sirius red. The cellular and stromal contents of the nodule were focally dis- 


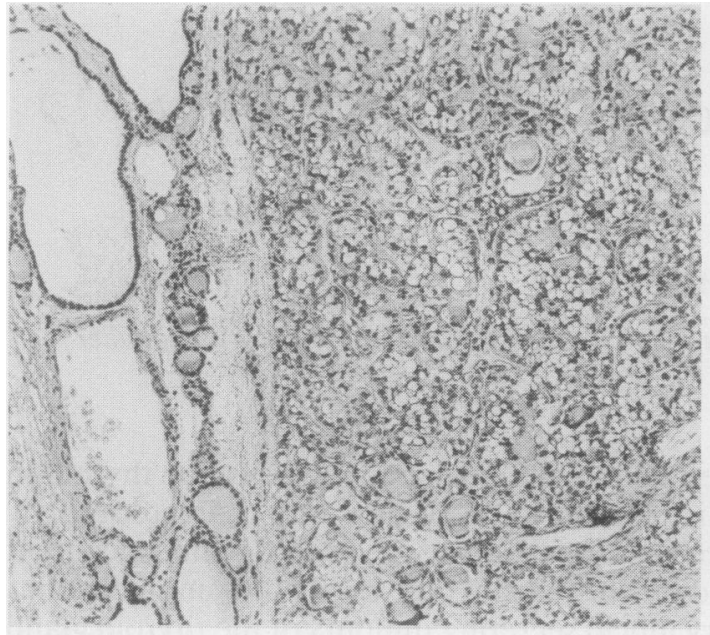

Fig. 1 Low power view of the mucin producing microfollicular adenoma (right) separated by a thin fibrous "capsule" from the neighbouring thyroid parenchyma (left). Haematoxylin and eosin $\times 140$.

rupted by areas of oedema and capillary haemorrhage. There was no evidence of capsular or vascular invasion in the multiple sections examined.

Immunocytochemical and mucin histochemical reactions were performed in one laboratory (Laboratoire d' anatomic pathologique, Institut Jean Godinot, Reims) in which these are routinely used.

\section{IMMUNOCYTOCHEMISTRY}

For immunostaining, the peroxidase-antiperoxidase method (PAP) was used. The following tumour markers were tested: thyroglobulin, calcitonin, and carcinoembryonic antigen (Dakopatts antisera).

Positive thyroglobulin immunostaining was noted in the vacuoles of most signet ring cells as well as in and around the colloid material within the small follicles (Fig. 3). Calcitonin and carcinoembryonic antigen antisera produced no visible reaction.

\section{MUCIN HISTOCHEMISTRY}

The following histochemical methods for mucin identification were used ${ }^{9}{ }^{10}$ : periodic acid Schiff reaction with and without diastase; Best's carmine; combined alcian blue, $\mathrm{pH} 2.5$, and periodic acid Schiff; combined high iron diamine and alcian blue, $\mathrm{pH} 2.5$; sialidase digestion using Vibrio cholerae sialidase followed by alcian blue, $\mathrm{pH} 2 \cdot 5$; periodic acid-sodium borohydride/saponification/periodic acid Schiff. Because of the fixation of the original material in Bouin's fluid, several normal controls fixed in both formol-saline and Bouin's fluid were also tested for comparison.

The vacuoles of the signet ring cells stained only

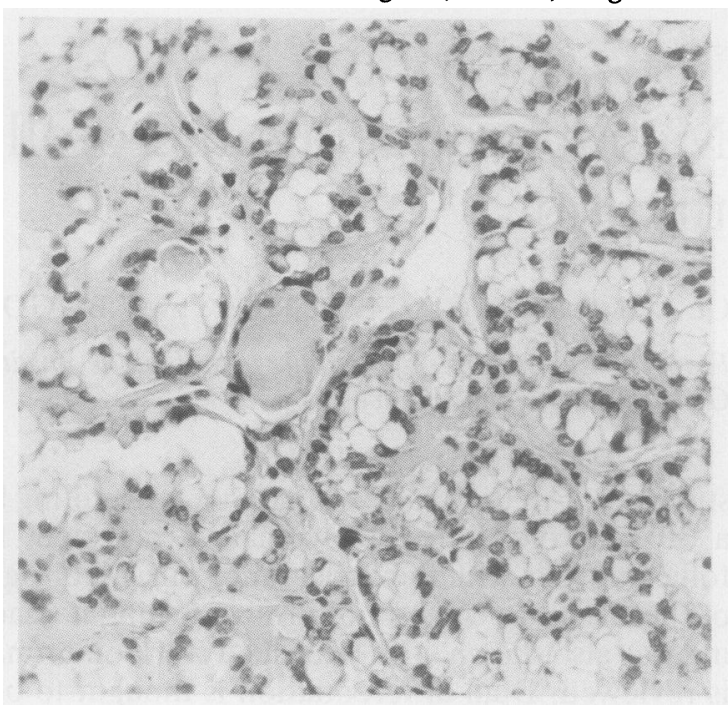

Fig. 2 High power field of Fig. 1 showing the nests of signet ring vacuolated cells with interspersed microfollicles containing colloid material. Haematoxylin and eosin $\times 350$.

blue with alcian blue and periodic acid Schiff and only brownish black with high iron diamine and alcian blue, indicating the presence of sulphomucins (Fig. 4); sialomucins and neutral mucins were not shown by the above two combined techniques. These vacuoles also contained scattered glycogen granules as shown by positive staining with periodic acid Schiff and Best's carmine but negative staining with D-periodic acid Schiff. The colloid material present in the small follicles stained densely magenta with periodic acid Schiff, with and without diastase, and with alcian blue. This colloid material was generally negative with the alcian blue and high iron diamine of both the alcian blue and periodic acid Schiff method and the high iron diamine and alcian blue method, but, in a few follicles only, there was a thin bluish (alcianophilic) rim around the periphery of the intrafollicular colloid material. Sialidase digestion followed by alcian blue, $\mathrm{pH} 2 \cdot 5$, and periodic acid-sodium borohydride/saponification/periodic acid Schiff were entirely negative, confirming the absence of either $\mathrm{N}$ - or $\mathrm{O}$-acetylated sialomucins in the epithelial components of the nodule.

Based on the results of histology, immunocytochemistry, and mucin histochemistry, a diagnosis of mucin producing benign microfollicular adenoma of the thyroid gland was made.

\section{Discussion}

Mucin production in primary benign or malignant neoplasms of the thyroid gland is extremely rare. ${ }^{1-8}$ The pathologist confronted with a solitary mucin 


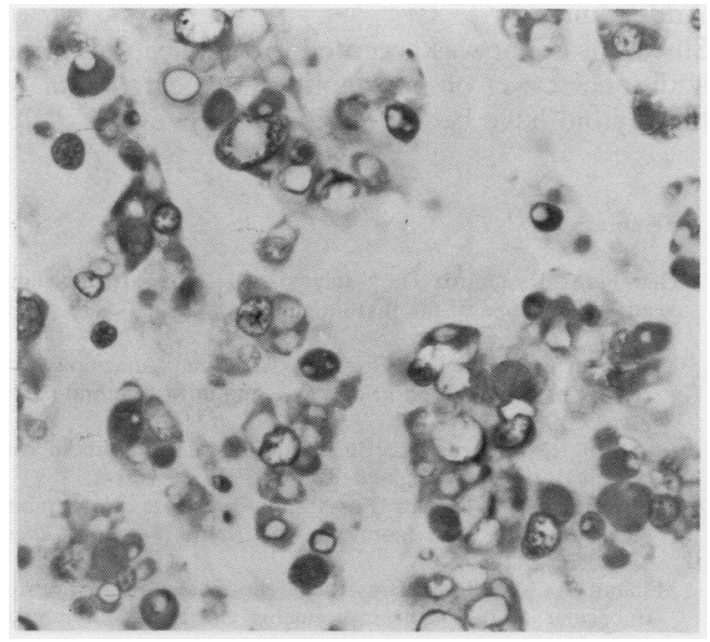

Fig. 3 Microphotograph showing the positive thyroglobulin immunostaining of the signet ring cells.

Peroxidase-antiperoxidase immunoperoxidase method. $\times$ 560.

secreting neoplasm within the thyroid must ask several questions. He should first try to eliminate the possibility of a metastasis from an extrathyroid site, an uncommon situation seen in disseminated malignant disease from bronchial, mammary, or renal carcinoma." Colonisation of thyroid adenomas by metastatic cancer cells has been recorded, ${ }^{12}$ but its incidence in practice is difficult to determine. The most important point is to be certain that the mucin producing neoplasm is of thyroid follicular epithelial origin and that it does not arise from some intrathyroid embryonic remnants such as the thyroglossal duct or ultimobranchial derivatives.

The published work on primary mucin producing tumours of the thyroid gland is scanty. Two cases of primary mucinous carcinoma ${ }^{12}$ and two cases of primary mucoepidermoid carcinoma ${ }^{34}$ have been reported, with ultrastructural study in two cases ${ }^{24}$ and biochemical tumour tissue assays in one case. ${ }^{4}$ Mucin production has also been reported in several cases of medullary carcinoma..$^{5-7}$ Recently, Mendelsohn $^{8}$ reported the first case of mucin secreting benign adenoma of the thyroid gland.

In the above reports ${ }^{1-8}$ the following histochemical methods for the demonstration of mucins were used: mucicarmine stain in all cases but one, ${ }^{5}$ periodic acid Schiff with and without diastase in six cases, ${ }^{2-68}$ alcian blue in three cases ${ }^{48}$ (though the $\mathrm{pH}$ of the alcian blue solution was indicated in only one instance), and colloidal iron in one case. ${ }^{8}$ Among the many techniques available today for the demonstration and identification of different

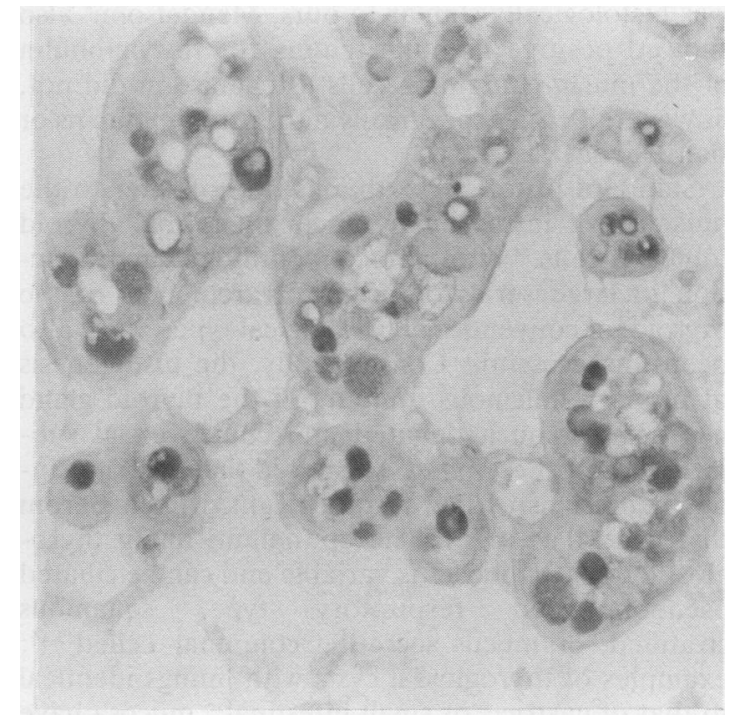

Fig. 4 Microphotograph showing brownish black staining of the vacuolar contents of the signet ring cells with high iron diamine indicating the presence of sulphomucins. High iron diamine and alcian blue $\times 560$.

mucins, ${ }^{91013}$ mucicarmine stain is still favoured by most North American authors. The problem is that mucicarmine will stain some but not all epithelial mucins. Although carboxylated mucins (sialomucins) or weakly acidic sulphated mucins (sulphomucins) give positive results with mucicarmine, neutral mucins and strongly-acidic sulphomucins are negative..$^{913}$ Apart from their value in demonstrating glycogen, conventional periodic acid Schiff with and without diastase cannot differentiate the different types of mucins.9 Alcian blue stains specifically all acidic mucins at $\mathrm{pH} 2.5$ but cannot separate sialomucins from sulphomucins. ${ }^{9}$ Equally, colloidal iron stains acidic mucins irrespective of their different types. ${ }^{9}$ Using the reliable combined techniques of alcian blue and periodic acid Schiff and high iron diamine and alcian blue, ${ }^{910}$ we have established the mucin profile of our case of mucin secreting adenoma of the thyroid as consisting specifically of sulphomucins with no evidence of either neutral mucins or sialomucins. To the best of our knowledge there are no published reports on the identification of sulphomucins as the sole mucin component of a primary mucinous neoplasm of the thyroid gland.

From the immunological viewpoint, we have also shown that the sulphomucin secreting cells of our case of adenoma showed positive immunostaining for thyroglobulin, therefore establishing their thyroid follicular epithelial origin. In his case report of a mucin producing adenoma of the thyroid which 
was histologically similar to ours, Mendelsohn ${ }^{8}$ also showed positive immunostaining for thyroglobulin of the mucin containing cells. Mendelsohn did not, however, identify specifically either sulphomucins or sialomucins.

Standard histology textbooks do not refer to the presence of intracellular mucin in normal thyroid follicular cells. ${ }^{14}$ Attempts to identify mucin production in large series of primary carcinomas of the thyroid, of conventional histological types, have also been unsuccessful.' Consequently, the histogenesis of primary mucinous tumours of the thyroid gland has been a much debated and controversial subject. ${ }^{1-8}$ Diaz-Perez et al ${ }^{1}$ suggested that such mucinous neoplasms of the thyroid might originate from thyroglossal vestiges. The epithelium lining thyroglossal cysts or sinuses is variable and can be ciliated pseudostratified respiratory type, squamous stratified, or mucus secreting columnar celled ${ }^{1516}$; examples of thyroglossal cysts with linings identical to that of gastric ${ }^{15}$ or small intestinal ${ }^{16}$ mucosa have been reported. Rhatigan $e a^{3}$ believed that salivary gland remnants trapped in the developing thyroid were responsible for their case of a mucoepidermoid carcinoma but these authors could not identify such remnants in the specimen.

A more likely explanation for the histogenesis of primary mucinous neoplasms of the thyroid could be dual differentiation. Dual differentiation such as the combination of mucin secretion and endocrine function has been increasingly reported in tumours arising from lung, pancreas, and gastrointestinal tract. ${ }^{17}$ We agree with Mendelsohn ${ }^{8}$ that dual differentiation could also apply to primary mucin secreting benign and malignant neoplasms of the thyroid gland, also an endodermal derived organ.

The finding of sulphomucins in a benign adenoma of the thyroid is remarkable, even on the basis of dual differentiation. Among the different types of mucins secreted by normal tissues, sulphomucins are predominantly found in the normal colonic mucosa. ${ }^{18}$

We believe that the detection and detailed profile of mucins, using alcian blue and periodic acid Schiff and high iron diamine and alcian blue coupled with immunocytochemistry, should now be studied in a large series of thyroid neoplasms. We have undertaken such an investigation.

We thank Professor ED Williams (Cardiff) for his critical review of the histological slides of this case and Dr M Dromer (St Quentin) and Dr MJ Delisles (Reims) for providing clinical data.

\section{Addendum}

Since this paper was accepted for publication, three additional cases of mucoepidermoid carcinoma of the thyroid have been described. ${ }^{19}$

\section{References}

' Diaz-Perez R, Quiroz H, Nishiyama RH. Primary mucinous adenocarcinoma of the thyroid gland. Cancer 1976;38:13235.

${ }^{2}$ Deligdisch L, Subhani Z, Gordon RE. Primary mucinous carcinoma of thyroid. Report of a case and ultrastructural study. Cancer 1980;45:2564-7.

${ }^{3}$ Rhatigan RM, Roque JL, Bucher RL. Mucoepidermoid carcinoma of the thyroid gland. Cancer 1977;39:210-4.

${ }^{4}$ Mizukami Y, Matsubara F, Hashimoto T, et al. Primary mucoepidermoid carcinoma in the thyroid gland. Cancer 1984;53:1741-5.

${ }^{5}$ Milhaud G, Calmettes C, Dreyfuss G, Moukhtar MS. An unusual trabecular thyroid cancer producing calcitonin. Experientia 1970;26:1381-3.

- Fernandes BJ, Berard YC, Rosen I. Mucus-producing medullary cell carcinoma of the thyroid gland. Am J Clin Pathol 1982;78:536-40.

7 Zaatari GS, Saigo PE, Huvos AG. Mucin production in medullary carcinoma of the thyroid. Arch Pathol Lab Med $1984 ; 107: 70-4$.

${ }^{8}$ Mendelsohn G. Signet-cell-simulating microfollicular adenoma of the thyroid. Am J Surg Pathol 1984;8:705-8.

${ }^{9}$ Cook HC. Carbohydrates. In: Bancroft JD, Stevens A, eds. Theory and practice of histological techniques. 2nd ed. Edinburgh: Churchill Livingstone, 1982:180-216.

${ }^{10}$ Filipe MI, Lake BD. Histochemistry in pathology. Edinburgh: Churchill Livingstone, 1983:310-3.

" Czech JM, Lichtor TR, Carney JA, Van Heerden JA. Neoplasms metastatic to the thyroid gland. Surg Gynecol Obstet 1982;155:503-5.

${ }^{12}$ Doniach I. The thyroid gland. In: Symmers WStC, ed. Systemic pathology. 2nd ed, vol 4. Edinburgh: Churchill Livingstone, 1978:2034.

${ }^{13}$ Drury RAB, Wallington EA. Carbohydrates and mucosubstances. In: Carleton's histological technique, 5th ed. Oxford: Oxford University Press, 1980:232-59.

${ }^{14}$ Tice LW. The thyroid gland. In: Weiss L, ed. Histology, cell and tissue biology. 5th ed. New York: McGraw-Hill, 1983:1090107.

is Willis RA. The borderland of embryology and pathology. 2nd ed. London: Butterworths, 1962:291-3.

${ }^{16}$ Meissner WA, Warren S. Tumors of the thyroid gland. In: Atlas of tumor pathology, 2nd series, fascicle 4. Washington DC: Armed Forces Institute of Pathology, 1969:28-9.

"Sidhu GS. The endodermal origin of digestive and respiratory tract APUD cells. Am J Pathol 1979;96:5-20.

${ }^{18}$ Filipe MI. Mucins in the human gastrointestinal epithelium: a review. Invest Cell Pathol 1979;2:195-216.

${ }^{14}$ Fransilla KO, Harach HR, Wasenius VM. Mucoepidermoid carcinoma of the thyroid. Histopathology 1984;8:847-60.

Requests for reprints to: Dr C Rigaud, Laboratoire d'anatomie pathologique, Institut Jean Godinot, BP 171, 51056 Reims Cedex, France. 